Document downloaded from:

http://hdl.handle.net/10251/149719

This paper must be cited as:

Ferreyra, DE.; Levis, F.; Thome, N. (2018). Revisiting the core EP inverse and its extension to rectangular matrices. Quaestiones Mathematicae. 41(2):265-281.

https://doi.org/10.2989/16073606.2017.1377779

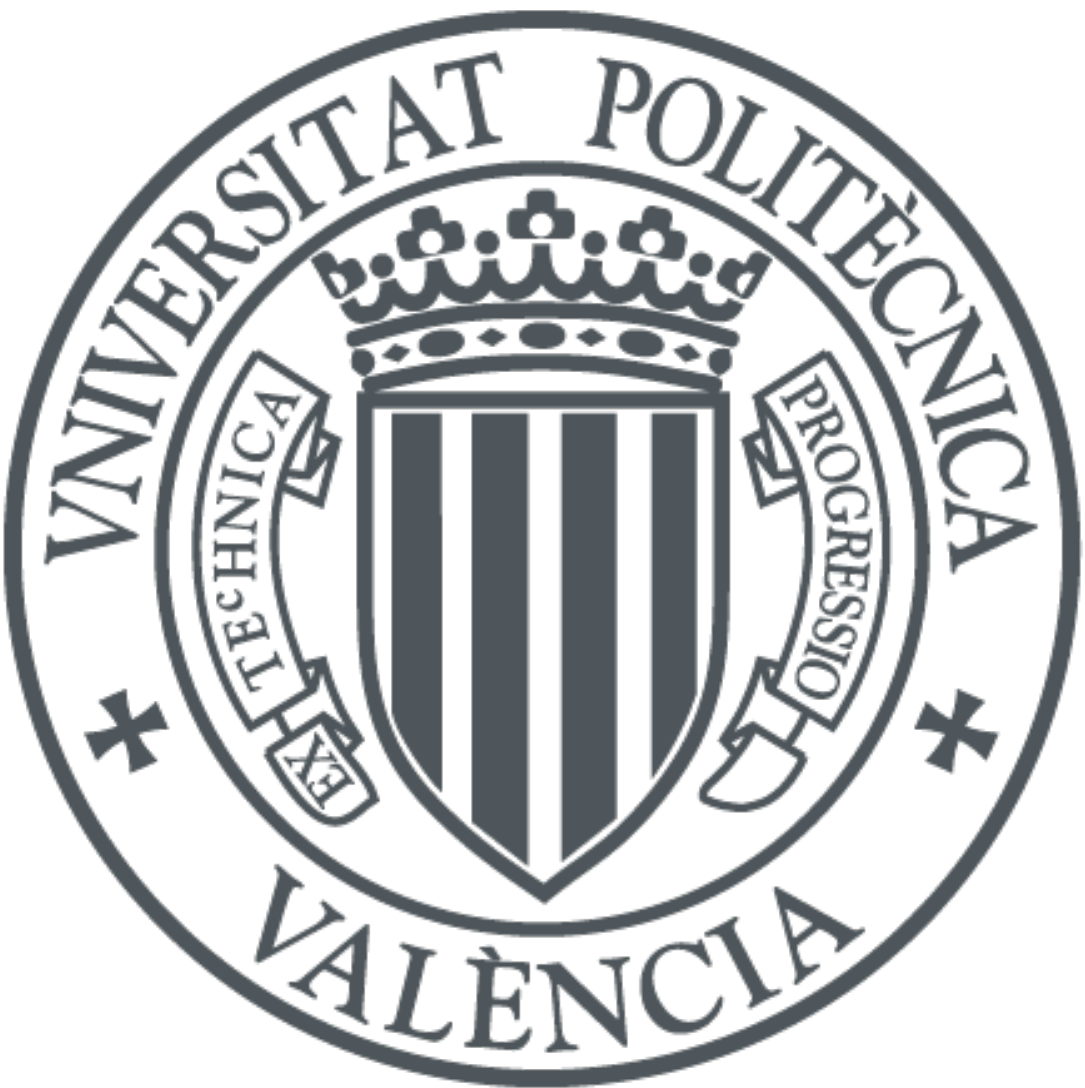

The final publication is available at

https://doi.org/10.2989/16073606.2017.1377779

Copyright Informa UK (National Inquiry Services Center)

Additional Information 


\title{
Revisiting the core EP inverse and its extension to rectangular matrices
}

\author{
D.E. Ferreyra* ${ }^{* \dagger}$, F.E. Levis ${ }^{\dagger}$, N. Thome ${ }^{\ddagger}$
}

\begin{abstract}
In this paper, we revise the core EP inverse of a square matrix introduced by Prasad and Mohana in [Core EP inverse, Linear and Multilinear Algebra, 62 (3) (2014) 792-802]. Firstly, we give a new representation and a new characterization of the core EP inverse. Then, we study some properties of the core EP inverse by using a representation by block matrices. Secondly, we extend the notion of core EP inverse to rectangular matrices by means of a weighted core EP decomposition. Finally, we study some properties of weighted core EP inverses.
\end{abstract}

AMS Classification: 15A09

Keywords: Generalized inverses, Moore-Penrose inverse, core inverse, generalized core inverse, core EP inverse, DMP generalized inverse.

\section{Introduction and background}

Let $\mathbb{C}^{m \times n}$ be the set of $m \times n$ complex matrices. For $A \in \mathbb{C}^{m \times n}$, the symbols $A^{*}, A^{-1}, \operatorname{rk}(A), \mathcal{N}(A)$, and $\mathcal{R}(A)$ will denote the conjugate transpose, the inverse $(m=n)$, the rank, the kernel and the range space of $A$, respectively. Moreover, $I_{n}$ will refer to the $n \times n$ identity matrix.

\footnotetext{
*Facultad de Ciencias Exactas y Naturales, Universidad Nacional de La Pampa, Av. Uruguay 151, Santa Rosa, 6300, La Pampa. Argentina.

${ }^{\dagger}$ Facultad de Ciencias Exactas Físico Químicas y Naturales, Universidad Nacional de Río Cuarto, RN 36 Km 601, Río Cuarto, 5800, Córdoba. Argentina. E-mail: deferreyra@exa.unrc.edu.ar, flevis@exa.unrc.edu.ar. Partially supported by a CONICET's Posdoctoral Research Fellowship, Universidad Nacional de Río Cuarto (grant PPI 18/C472) and CONICET (grant PIP 112-201501-00433CO).

${ }^{\ddagger}$ Instituto Universitario de Matemática Multidisciplinar, Universitat Politècnica de València, 46022, Valencia, Spain. E-mail: njthome@mat.upv.es. Partially supported by Ministerio de Economía y Competitividad of Spain (grants DGI MTM2013-43678-P and Red de Excelencia MTM2015-68805-REDT).
} 
Let $A \in \mathbb{C}^{m \times n}$. We recall that the unique matrix $X \in \mathbb{C}^{n \times m}$ satisfying

$$
A X A=A, \quad X A X=X, \quad(A X)^{*}=A X, \quad \text { and } \quad(X A)^{*}=X A
$$

is called the Moore-Penrose inverse of $A$ and is denoted by $A^{\dagger}$. A matrix $X \in \mathbb{C}^{n \times m}$ that satisfies the only equality $A X A=A$ is called an inner inverse of $A$ and is denoted by $A^{-}$; and a matrix $X \in \mathbb{C}^{n \times m}$ that satisfies the only equality $X A X=X$ is called an outer inverse of $A$. The class of all inner inverses of $A$ will be denoted by $A\{1\}$.

For a given complex square matrix $A$, the index of $A$, denoted by $\operatorname{Ind}(A)$, is the smallest nonnegative integer $k$ such that $\mathcal{R}\left(A^{k}\right)=\mathcal{R}\left(A^{k+1}\right)$. We observe that the index of a nonsingular matrix $A$ is 0 , and by convention, the index of the null matrix is 1 . We also recall that the Drazin inverse of $A \in \mathbb{C}^{n \times n}$ is the unique matrix $X \in \mathbb{C}^{n \times n}$ such that $X A X=X, A X=X A$, and $A^{k+1} X=A^{k}$, where $k=\operatorname{Ind}(A)$, and is denoted by $A^{d}$. If $A \in \mathbb{C}^{n \times n}$ satisfies $\operatorname{Ind}(A) \leq 1$, then the Drazin inverse of $A$ is called the group inverse of $A$ and is denoted by $A^{\#}$.

In [2], Baksalary and Trenkler introduced a new generalized inverse in the following way for a given matrix $A \in \mathbb{C}^{n \times n}$. A matrix $X \in \mathbb{C}^{n \times n}$ satisfying

$$
A X=P_{A} \text { and } \mathcal{R}(X) \subseteq \mathcal{R}(A)
$$

is called the core inverse of $A$ and is denoted by $A^{\boxplus}$, where $P_{A}$ is the orthogonal projector onto the range of $A$, i.e., $P_{A}=A A^{\dagger}$. Moreover, it was proved that $A$ is core invertible if and only if $\operatorname{Ind}(A) \leq 1$.

Three generalizations of the core inverse were recently introduced for $n \times n$ complex matrices, namely core EP inverses, BT inverses, and DMP inverses. In order to recall these concepts we assume that $\operatorname{Ind}(A)=k$ for a given matrix $A \in \mathbb{C}^{n \times n}$. Firstly, the unique matrix $X \in \mathbb{C}^{n \times n}$ such that

$$
X A X=X \quad \text { and } \quad \mathcal{R}(X)=\mathcal{R}\left(X^{*}\right)=\mathcal{R}\left(A^{k}\right),
$$

is called the core EP inverse of $A$ and is denoted by $A^{\oplus}[12]$. Secondly, the concept of BT inverse of $A$ was introduced in [3] and originally referred as generalized core inverse. In this case, the matrix

$$
A^{\diamond}:=\left(A P_{A}\right)^{\dagger}
$$

is called the BT inverse of $A$. Thirdly, another generalization of the core inverse was given in [10]. The unique matrix $X \in \mathbb{C}^{n \times n}$ satisfying

$$
X A X=X, \quad X A=A^{d} A, \quad \text { and } \quad A^{k} X=A^{k} A^{\dagger},
$$

is called the DMP inverse of $A$ and is denoted by $A^{d, \dagger}$. For some related results we refer the reader to $[5,9,11]$. 
This paper is organized as follows. In Section 2, we give a new necessary and sufficient condition for a matrix to be the core EP inverse, and we state some properties of them. In Section 3, we establish a canonical form for the core EP inverse by using the Hartwig-Spindelböck decomposition. Then, we derive some properties of the core EP inverse by using this new representation. In Section 4, we obtain a simultaneous unitarily triangularization of a pair of rectangular matrices which extends the core EP decomposition from square to rectangular matrices. In Section 5, we extend the notion of core EP inverse to rectangular matrices and we study some properties of them.

\section{Revisiting the core EP inverse}

In [12], the following result was discussed in the case of matrices over a field.

Lemma 2.1. [12, Theorem 3.5] For a given matrix $A \in \mathbb{C}^{n \times n}$ with $\operatorname{Ind}(A)=k$, its core EP inverse $A^{\oplus}$ always exists and it is unique. Further, the core EP inverse is given by

$$
A^{\oplus}=A^{k}\left(\left(A^{*}\right)^{k} A^{k+1}\right)^{\dagger}\left(A^{*}\right)^{k} .
$$

According to Theorem 2.2 in [13], every matrix $A \in \mathbb{C}^{n \times n}$ with $\operatorname{Ind}(A)=k$ can be represented in the form

$$
A=(A)_{1}+(A)_{2}, \quad(A)_{1}:=U\left[\begin{array}{cc}
T & S \\
0 & 0
\end{array}\right] U^{*}, \quad(A)_{2}:=U\left[\begin{array}{cc}
0 & 0 \\
0 & N
\end{array}\right] U^{*}
$$

where $T$ is nonsingular with $\operatorname{rk}(T)=\operatorname{rk}\left(A^{k}\right), N$ is nilpotent of index $k$, and $U$ is unitary. The representation of $A$ given in (4) satisfies $\operatorname{Ind}\left((A)_{1}\right) \leq 1,\left((A)_{2}\right)^{k}=0$ and $(A)_{1}^{*}(A)_{2}=(A)_{2}(A)_{1}=0[13$, Theorem 2.1]. Moreover, it is unique [13, Theorem 2.4] and is called the core EP decomposition of $A$.

Lemma 2.2. [13, Theorem 3.2] Let $A \in \mathbb{C}^{n \times n}$ be written as in (4) such that $\operatorname{Ind}(A)=k$. Then $A^{\oplus}=(A)_{1}^{\oplus}$. Furthermore,

$$
A^{\oplus}=U\left[\begin{array}{cc}
T^{-1} & 0 \\
0 & 0
\end{array}\right] U^{*} \text {. }
$$

The lemma below gives a characterization of the core EP inverse which is included in Lemma 3.3 given in [12].

Lemma 2.3. Let $A, X \in \mathbb{C}^{n \times n}$ be such that $\operatorname{Ind}(A)=k$. Then $X$ is the core EP of $A$ if and only if $X$ satisfies the conditions:

$$
X A^{k+1}=A^{k}, \quad X A X=X, \quad(A X)^{*}=A X, \quad \text { and } \quad \mathcal{R}(X) \subseteq \mathcal{R}\left(A^{k}\right)
$$


Remark 2.4. According to [12, Lemma 3.3], we observe that Lemma 2.3 remains valid whether the equation $X A^{k+1}=A^{k}$ is replaced by $X A^{k+2}=A^{k+1}$, since $\mathcal{R}\left(A^{k}\right)=\mathcal{R}\left(A^{k+1}\right)$ when $\operatorname{Ind}(A)=k$.

In the following lemma we compute the projector $P_{A^{\ell}}$ from the expression of $A$ given in (4).

Lemma 2.5. Let $A \in \mathbb{C}^{n \times n}$ be written as in (4) such that $\operatorname{Ind}(A)=k$. Then, for each integer $\ell \geq k$,

$$
P_{A^{\ell}}=U\left[\begin{array}{cc}
I_{r k\left(A^{k}\right)} & 0 \\
0 & 0
\end{array}\right] U^{*}
$$

Proof. If we write $A$ as in (4) then

$$
A^{\ell}=U\left[\begin{array}{cc}
T^{\ell} & \widetilde{T} \\
0 & N^{\ell}
\end{array}\right] U^{*}=U\left[\begin{array}{cc}
T^{\ell} & \widetilde{T} \\
0 & 0
\end{array}\right] U^{*},
$$

where $\widetilde{T}=\sum_{j=0}^{\ell} T^{j} S N^{\ell-j}$. On the other hand, since for $\ell \geq k$,

$$
\operatorname{rk}\left(\left[\begin{array}{cc}
T^{\ell} & \widetilde{T}
\end{array}\right]\left[\begin{array}{c}
\left(T^{\ell}\right)^{*} \\
\widetilde{T}^{*}
\end{array}\right]\right)=\operatorname{rk}\left(\left[\begin{array}{cc}
T^{\ell} & \widetilde{T}
\end{array}\right]\right)=\operatorname{rk}(T)=\operatorname{rk}\left(A^{k}\right)=\operatorname{rk}\left(A^{\ell}\right),
$$

we have that $T^{\ell}\left(T^{\ell}\right)^{*}+\widetilde{T} \widetilde{T}^{*}$ is nonsingular. Therefore, by [8, Lemma 1$]$ we get

$$
\left(A^{\ell}\right)^{\dagger}=U\left[\begin{array}{cc}
\left(T^{\ell}\right)^{*}\left[T^{\ell}\left(T^{\ell}\right)^{*}+\widetilde{T} \widetilde{T}^{*}\right]^{-1} & 0 \\
(\widetilde{T})^{*}\left[T^{\ell}\left(T^{\ell}\right)^{*}+\widetilde{T} \widetilde{T}^{*}\right]^{-1} & 0
\end{array}\right] U^{*}
$$

Now,

$$
P_{A^{\ell}}=A^{\ell}\left(A^{\ell}\right)^{\dagger}=U\left[\begin{array}{cc}
I_{\mathrm{rk}\left(A^{k}\right)} & 0 \\
0 & 0
\end{array}\right] U^{*}
$$

Next, we get a simple necessary and sufficient condition for $A$ to be the core EP inverse. Before that, we present a lemma of uniqueness.

Lemma 2.6. Let $A \in \mathbb{C}^{n \times n}$ be such that $\operatorname{Ind}(A)=k$. If there exists $X \in \mathbb{C}^{n \times n}$ such that

$$
A X=P_{A^{k}} \quad \text { and } \quad \mathcal{R}(X) \subseteq \mathcal{R}\left(A^{k}\right)
$$

then it is unique.

Proof. Assume that $X_{1}$ and $X_{2}$ satisfy (7), that is $A X_{1}=A X_{2}=A^{k}\left(A^{k}\right)^{\dagger}, \mathcal{R}\left(X_{1}\right) \subseteq \mathcal{R}\left(A^{k}\right)$, and $\mathcal{R}\left(X_{2}\right) \subseteq \mathcal{R}\left(A^{k}\right)$. Since $A\left(X_{1}-X_{2}\right)=0$, we obtain $\mathcal{R}\left(X_{1}-X_{2}\right) \subseteq \mathcal{N}(A) \subseteq \mathcal{N}\left(A^{k}\right)$. We also get that $\mathcal{R}\left(X_{1}-X_{2}\right) \subseteq \mathcal{R}\left(A^{k}\right)$. Therefore, $\mathcal{R}\left(X_{1}-X_{2}\right) \subseteq \mathcal{N}\left(A^{k}\right) \cap \mathcal{R}\left(A^{k}\right)=\{0\}$ because $A$ has index $k$. Thus, $X_{1}=X_{2}$. 
Theorem 2.7. Let $A, X \in \mathbb{C}^{n \times n}$ be such that $\operatorname{Ind}(A)=k$. Then $X$ is the core EP of $A$ if and only if $X$ satisfies (7). In this case, we have $A^{\oplus}=X=\left(A P_{A^{k}}\right)^{\dagger}$.

Proof. Let $A$ be written as in the form (4). Suppose that $X$ is the core EP inverse of $A$. Lemma 2.2 implies that

$$
X=U\left[\begin{array}{cc}
T^{-1} & 0 \\
0 & 0
\end{array}\right] U^{*}
$$

Consequently, Lemma 2.5 yields

$$
A X=U\left[\begin{array}{cc}
I_{\operatorname{rk}\left(A^{k}\right)} & 0 \\
0 & 0
\end{array}\right] U^{*}=P_{A^{k}}
$$

According to Lemma 2.3, we have that $\mathcal{R}(X) \subseteq \mathcal{R}\left(A^{k}\right)$ holds.

In order to establish the sufficiency, by Lemma 2.6 we have that the matrix $X$ in (8) is the unique matrix that satisfies (7). Now, Lemma 2.2 implies that $X$ is the core EP inverse of $A$. Finally, from (4) and (6) it follows that

$$
A P_{A^{k}}=U\left[\begin{array}{cc}
T & 0 \\
0 & 0
\end{array}\right] U^{*}
$$

and, hence, $\left(A P_{A^{k}}\right)^{\dagger}=X$ by [4, pp. 49].

From [2, Theorem 1] it is known that $A^{\oplus}$ belong to $A\{1\}$. It is of interest to inquire whether $A^{\oplus}$ belongs to $A\{1\}$ as well.

Theorem 2.8. Let $A \in \mathbb{C}^{n \times n}$. The following conditions are equivalent.

(i) $A^{\oplus} \in A\{1\}$,

(ii) $\operatorname{Ind}(A) \leq 1$,

(iii) $A^{\diamond} \in A\{1\}$.

Moreover, in this case, $A^{\oplus}=A^{\diamond}=A^{\boxplus}$.

Proof. Suppose $A$ has the form (4). From Theorem 2.7 we have $A^{\oplus} \in A\{1\}$ if and only if $A=$ $A A^{\oplus} A=P_{A^{k}} A$. This condition is equivalent to

$$
U\left[\begin{array}{cc}
T & S \\
0 & N
\end{array}\right] U^{*}=U\left[\begin{array}{cc}
I_{\operatorname{rk}\left(A^{k}\right)} & 0 \\
0 & 0
\end{array}\right]\left[\begin{array}{cc}
T & S \\
0 & N
\end{array}\right] U^{*},
$$

by Lemma 2.5. Thus, we arrive at $A^{\oplus} \in A\{1\}$ if and only if $N=0$, i.e., $A=(A)_{1}$ holds. This shows that (i) and (ii) are equivalent. The equivalence between (ii) and (iii) was proved in [3, Theorem 2]. 
If $\operatorname{Ind}(A) \leq 1$, by Lemma 2.5, Theorem 2.7 and $[2$, Theorem 1 (iii)] we obtain

$$
A=U\left[\begin{array}{cc}
T & S \\
0 & 0
\end{array}\right] U^{*} \quad \text { and } \quad A^{\diamond}=\left(A P_{A}\right)^{\dagger}=U\left[\begin{array}{cc}
T^{-1} & 0 \\
0 & 0
\end{array}\right] U^{*}=A^{\oplus}=A^{\boxplus} .
$$

However, the equality $A^{\oplus}=A^{\diamond}$ does not imply that $\operatorname{Ind}(A) \leq 1$ holds, as the following example shows. If we take

$$
A=\left[\begin{array}{llll}
1 & 1 & 0 & 0 \\
0 & 0 & 0 & 0 \\
0 & 0 & 0 & 1 \\
0 & 0 & 0 & 0
\end{array}\right],
$$

we have that

$$
A^{\oplus}=A^{\diamond}=\left[\begin{array}{llll}
1 & 0 & 0 & 0 \\
0 & 0 & 0 & 0 \\
0 & 0 & 0 & 0 \\
0 & 0 & 0 & 0
\end{array}\right]
$$

but $\operatorname{Ind}(A)=2$.

We recall that a square complex matrix $A$ is said to be EP if $A$ and its conjugate transpose $A^{*}$ have the same range. Theorem 1 in [2] also asserts that $\left(A^{\boxplus}\right) \oplus=A P_{A}$ and $A^{\boxplus}$ is necessarily EP. In the following result we show that these statements remain valid when the superscript $\circledast$ is replaced with $\oplus$.

Theorem 2.9. Let $A \in \mathbb{C}^{n \times n}$ be such that $\operatorname{Ind}(A)=k$. The following statements hold:

(i) $A^{\oplus}$ is $E P$;

(ii) $A\left(A^{\oplus}\right)^{2}=A^{\oplus}$;

(iii) $\left(A^{\oplus}\right)^{\oplus}=A P_{A^{k}}$;

(iv) $A P_{A^{k}}$ is $E P$.

Proof. Clearly (i) follows from definition of the core EP inverse. On other hand, by (4)-(7) it is easy to check that the conditions (ii)-(iv) are valid.

Remark 2.10. A similar result to Theorem 2.9 can be found in [3] for BT inverses. 


\section{A canonical form for core EP inverses}

In this section we give a canonical form for the core EP inverse of a square matrix $A$ by using the Hartwig-Spindelböck decomposition [7, Corollary 6]. For any matrix $A \in \mathbb{C}^{n \times n}$ of rank $r>0$ the Hartwig-Spindelböck decomposition is given by

$$
A=U\left[\begin{array}{cc}
\Sigma K & \Sigma L \\
0 & 0
\end{array}\right] U^{*},
$$

where $U \in \mathbb{C}^{n \times n}$ is unitary, $\Sigma=\operatorname{diag}\left(\sigma_{1} I_{r_{1}}, \sigma_{2} I_{r_{2}} \cdots, \sigma_{t} I_{r_{t}}\right)$ is a diagonal matrix, the diagonal entries $\sigma_{i}$ being singular values of $A, \sigma_{1}>\sigma_{2}>\cdots>\sigma_{t}>0, r_{1}+r_{2}+\cdots+r_{t}=r$ and $K \in \mathbb{C}^{r \times r}, L \in \mathbb{C}^{r \times(n-r)}$ satisfy $K K^{*}+L L^{*}=I_{r}$.

Now, we can derive the core EP inverse of $A$ of index $k$ for which we need the following result that is a particular case of Corollary given in [6, pp. 365].

Corollary 3.1. Let $A \in \mathbb{C}^{m \times n}$. If $A Q$ is a product of matrices for which there exists a matrix $Q^{\prime}$ such that $A Q Q^{\prime}=A$ then $A Q(A Q)^{*}+I_{m}-A A^{\dagger}$ is nonsingular and

$$
(A Q)^{\dagger}=(A Q)^{*}\left[A Q(A Q)^{*}+I_{m}-A A^{\dagger}\right]^{-1} .
$$

Theorem 3.2. Let $A \in \mathbb{C}^{n \times n}$ be written as in (9). Then

$$
A^{\oplus}=U\left[\begin{array}{cc}
(\Sigma K)^{\oplus} & 0 \\
0 & 0
\end{array}\right] U^{*} .
$$

Proof. Let $\operatorname{Ind}(A)=k$. By Theorem 2.7, we have $A^{\oplus}=\left(A P_{A^{k}}\right)^{\dagger}=\left(A A^{k}\left(A^{k}\right)^{\dagger}\right)^{\dagger}$. If we suppose that $A$ is written as in (9) then

$$
A^{k}=U\left[\begin{array}{cc}
(\Sigma K)^{k} & (\Sigma K)^{k-1} \Sigma L \\
0 & 0
\end{array}\right] U^{*}
$$

It follows that

$$
\left(A^{k}\right)^{\dagger}=U\left[\begin{array}{cc}
(\Sigma K)^{k} & (\Sigma K)^{k-1} \Sigma L \\
0 & 0
\end{array}\right]^{\dagger} U^{*}
$$

and applying $[8$, Lemma 1] to (11) we obtain

$$
\left(A^{k}\right)^{\dagger}=U\left[\begin{array}{cc}
P^{*} R^{\dagger} & 0 \\
Q^{*} R^{\dagger} & 0
\end{array}\right] U^{*},
$$

where $R=P P^{*}+Q Q^{*}, P=(\Sigma K)^{k}$ and $Q=(\Sigma K)^{k-1} \Sigma L$. This implies that

$$
P_{A^{k}}=A^{k}\left(A^{k}\right)^{\dagger}=U\left[\begin{array}{cc}
R R^{\dagger} & 0 \\
0 & 0
\end{array}\right] U^{*} .
$$


Now, we calculate $R$ as follows

$$
\begin{aligned}
R & =\left[\begin{array}{ll}
P & Q
\end{array}\right]\left[\begin{array}{ll}
P & Q
\end{array}\right]^{*} \\
& =\left[\begin{array}{ll}
(\Sigma K)^{k} & (\Sigma K)^{k-1} \Sigma L
\end{array}\right]\left[\begin{array}{l}
\left.K^{*}(\Sigma K)^{k-1} \Sigma\right)^{*} \\
L^{*}\left((\Sigma K)^{k-1} \Sigma\right)^{*}
\end{array}\right] \\
& =(\Sigma K)^{k-1} \Sigma\left[\begin{array}{ll}
K & L
\end{array}\right]\left[\begin{array}{c}
K^{*} \\
L^{*}
\end{array}\right]\left((\Sigma K)^{k-1} \Sigma\right)^{*} \\
& =(\Sigma K)^{k-1} \Sigma\left((\Sigma K)^{k-1} \Sigma\right)^{*} .
\end{aligned}
$$

On the other hand, we know that $B^{\dagger}=B^{*}\left(B B^{*}\right)^{\dagger}$ for any complex matrix $B$. In consequence, from (13) we get

$$
R R^{\dagger}=(\Sigma K)^{k-1} \Sigma\left((\Sigma K)^{k-1} \Sigma\right)^{\dagger} .
$$

If we set $M=(\Sigma K)^{k-1}$, by using Corollary 3.1 with $Q=\Sigma$ and $Q^{\prime}=\Sigma^{-1}$ we have

$$
R R^{\dagger}=M \Sigma(M \Sigma)^{*}\left[M \Sigma(M \Sigma)^{*}+I_{r}-M M^{\dagger}\right]^{-1} .
$$

Since $M=M M^{\dagger} M$ and $M M^{\dagger}$ is a projector, then

$$
M \Sigma(M \Sigma)^{*}=M M^{\dagger} M \Sigma(M \Sigma)^{*}+M M^{\dagger}-\left(M M^{\dagger}\right)^{2}=M M^{\dagger}\left[M \Sigma(M \Sigma)^{*}+I_{r}-M M^{\dagger}\right] .
$$

Hence, from (14) we obtain

$$
R R^{\dagger}=M M^{\dagger}=(\Sigma K)^{k-1}\left((\Sigma K)^{k-1}\right)^{\dagger}
$$

Now, (12) and (15) imply

$$
P_{A^{k}}=U\left[\begin{array}{cc}
(\Sigma K)^{k-1}\left((\Sigma K)^{k-1}\right)^{\dagger} & 0 \\
0 & 0
\end{array}\right] U^{*} .
$$

So, by [4, pp. 49] and [8, Lemma 1] it follows that

$$
\begin{aligned}
A^{\oplus}=\left(A P_{A^{k}}\right)^{\dagger} & =\left[U\left[\begin{array}{cc}
\Sigma K & \Sigma L \\
0 & 0
\end{array}\right]\left[\begin{array}{cc}
(\Sigma K)^{k-1}\left((\Sigma K)^{k-1}\right)^{\dagger} & 0 \\
0 & 0
\end{array}\right] U^{*}\right]^{\dagger} . \\
& =U\left[\begin{array}{cc}
\Sigma K(\Sigma K)^{k-1}\left((\Sigma K)^{k-1}\right)^{\dagger} & 0 \\
0 & 0
\end{array}\right]^{\dagger} U^{*} . \\
& =U\left[\begin{array}{cc}
\left(\Sigma K(\Sigma K)^{k-1}\left((\Sigma K)^{k-1}\right)^{\dagger}\right)^{\dagger} & 0 \\
0 & 0
\end{array}\right] U^{*} .
\end{aligned}
$$


It is well known [10, Lemma 2.8] that the matrix $\Sigma K$ has index $k-1$. In consequence, by Theorem 2.7 we get

$$
(\Sigma K)^{\oplus}=\left(\Sigma K P_{(\Sigma K)^{k-1}}\right)^{\dagger}=\left(\Sigma K(\Sigma K)^{k-1}\left((\Sigma K)^{k-1}\right)^{\dagger}\right)^{\dagger}
$$

Hence, (10) holds.

From [3, Lemma 2] and [10, Theorem 2.5], if $A$ has the form in (9), we have

$$
A^{\diamond}=U\left[\begin{array}{cc}
(\Sigma K)^{\dagger} & 0 \\
0 & 0
\end{array}\right] U^{*} \text { and } A^{d, \dagger}=U\left[\begin{array}{cc}
(\Sigma K)^{d} & 0 \\
0 & 0
\end{array}\right] U^{*},
$$

respectively. Now, we can state the following consequences.

Corollary 3.3. Let $A \in \mathbb{C}^{n \times n}$ be written as in (9). Then the following statements are equivalent:

(i) $A^{\oplus}=A^{\diamond}$;

(ii) $A^{d, \dagger}=A^{\diamond}$;

(iii) $\Sigma K$ is $E P$.

Moreover, in this case, $\operatorname{Ind}(A) \leq 2$.

Proof. From (10) and (16), it is clear that (i) and (ii) are equivalent to $(\Sigma K)^{\oplus}=(\Sigma K)^{\dagger}$ and $(\Sigma K)^{d}=$ $(\Sigma K)^{\dagger}$, respectively. By item (i) of Theorem 2.9, the former of these conditions is equivalent to the assertion $(\Sigma K)^{\dagger}$ is EP, which is equivalent to the fact that $\Sigma K$ is EP. On the other hand, from [4, Theorem 4, pp. 157] $(\Sigma K)^{d}=(\Sigma K)^{\dagger}$ if only if $\Sigma K$ is EP. Finally, that $\operatorname{Ind}(A) \leq 2$ is a necessary condition in this case follows from (iii) and [10, Lemma 2.8].

Corollary 3.4. Let $A \in \mathbb{C}^{n \times n}$ be written as in (9). Then $A^{\oplus}=A^{d, \dagger}$ if and only if $(\Sigma K)^{\oplus}=(\Sigma K)^{d}$.

We notice that, in general, core EP, BT, and DMP inverses are all different each other as the following example shows.

Example 3.5. We consider the matrix

$$
A=\left[\begin{array}{rrr}
1 & 1 & -1 \\
1 & 0 & 2 \\
2 & 1 & 1
\end{array}\right]
$$

It is easy to check that $\operatorname{Ind}(A)=2$,

$$
A^{\oplus}=\left[\begin{array}{ccc}
0 & 0 & 0 \\
0 & \frac{1}{4} & \frac{1}{4} \\
0 & \frac{1}{4} & \frac{1}{4}
\end{array}\right], \quad A^{\diamond}=\left[\begin{array}{ccc}
0 & \frac{1}{7} & \frac{1}{7} \\
0 & \frac{1}{28} & \frac{1}{28} \\
0 & \frac{5}{28} & \frac{5}{28}
\end{array}\right], \quad \text { and } \quad A^{d, \dagger}=\left[\begin{array}{ccc}
0 & 0 & 0 \\
\frac{1}{3} & \frac{1}{12} & \frac{5}{12} \\
\frac{1}{3} & \frac{1}{12} & \frac{5}{12}
\end{array}\right] \text {. }
$$


Corollary 3.6. Let $A \in \mathbb{C}^{n \times n}$ be written as in (9) and $A^{\oplus}$ expressed as in (10). Then

(i) $\left(A^{\oplus}\right)^{\dagger}=U\left[\begin{array}{cc}\left((\Sigma K)^{\oplus}\right)^{\dagger} & 0 \\ 0 & 0\end{array}\right] U^{*}$;

(ii) $\left(A^{\oplus}\right)^{\#}=U\left[\begin{array}{cc}\left((\Sigma K)^{\oplus}\right)^{\#} & 0 \\ 0 & 0\end{array}\right] U^{*}$;

(iii) $\left(A^{\oplus}\right)^{\oplus}=U\left[\begin{array}{cc}\left((\Sigma K)^{\oplus}\right)^{\oplus} & 0 \\ 0 & 0\end{array}\right] U^{*}$.

Proof. (i) It is a direct application of Theorem 3.2.

(ii) By item (i) of Theorem 2.9, $A^{\oplus}$ and $(\Sigma K)^{\oplus}$ are EP matrices. According to [4, Theorem 4, pp. 157] we have $\left(A^{\oplus}\right)^{\#}=\left(A^{\oplus}\right)^{\dagger}$ and $\left((\Sigma K)^{\oplus}\right)^{\#}=\left((\Sigma K)^{\oplus}\right)^{\dagger}$. So, (ii) follows from (i).

(iii) From (10) and (i) it is straightforward to obtain

$$
A^{\oplus} A^{\oplus}\left(A^{\oplus}\right)^{\dagger}=U\left[\begin{array}{cc}
(\Sigma K)^{\oplus}(\Sigma K)^{\oplus}\left((\Sigma K)^{\oplus}\right)^{\dagger} & 0 \\
0 & 0
\end{array}\right] U^{*} .
$$

Since $A^{\oplus}$ and $(\Sigma K)^{\oplus}$ are EP then both matrices have its index at most 1 . Therefore, by Theorem 2.7 and $[8$, Lemma 1] we have

$\left(A^{\oplus}\right)^{\oplus}=\left(A^{\oplus} A^{\oplus}\left(A^{\oplus}\right)^{\dagger}\right)^{\dagger}=U\left[\begin{array}{cc}\left((\Sigma K)^{\oplus}(\Sigma K)^{\oplus}\left((\Sigma K)^{\oplus}\right)^{\dagger}\right)^{\dagger} & 0 \\ 0 & 0\end{array}\right] U^{*}=U\left[\begin{array}{cc}\left((\Sigma K)^{\oplus}\right)^{\oplus} & 0 \\ 0 & 0\end{array}\right] U^{*}$.

Theorem 3.7. Let $A \in \mathbb{C}^{n \times n}$ be such that $\operatorname{Ind}(A)=k$. Then:

(i) $A A^{\oplus}$ is the orthogonal projector onto the column space of $A^{k}$,

(ii) $A^{\oplus} A$ is the oblique projector onto the column space of $A^{k}$ along the null space of $\left(A^{k+1}\right)^{*} A$.

Proof. (i) By Theorem 2.7 we obtain $A A^{\oplus}=P_{A^{k}}=A^{k}\left(A^{k}\right)^{\dagger}$ which is clearly an orthogonal projector onto $\mathcal{R}\left(A^{k}\right)$ (see [1, pp. 2814]).

(ii) Since by definition $A^{\oplus}$ is an outer inverse of $A, A^{\oplus} A$ is idempotent, thus $A^{\oplus} A$ is an oblique projector. Moreover, from $\mathcal{R}\left(A^{\oplus}\right)=\mathcal{R}\left(A^{k}\right)$ it follows that $\mathcal{R}\left(A^{\oplus} A\right)=\mathcal{R}\left(A^{\oplus}\right)=\mathcal{R}\left(A^{k}\right)$. On the other hand, we are going to prove that $\mathcal{N}\left(A^{\oplus} A\right)=\mathcal{N}\left(\left(A^{k+1}\right)^{*} A\right)$ holds. In fact, if $x \in \mathcal{N}\left(A^{\oplus} A\right)=$ $\mathcal{N}\left(\left(A P_{A^{k}}\right)^{\dagger} A\right)$ then $A x \in \mathcal{N}\left(\left(A P_{A^{k}}\right)^{\dagger}\right)=\mathcal{N}\left(\left(A P_{A^{k}}\right)^{*}\right)$. Thus,

$$
\mathcal{N}\left(A^{\oplus} A\right) \subseteq \mathcal{N}\left(\left(A P_{A^{k}}\right)^{*} A\right) \subseteq \mathcal{N}\left(\left(A^{k}\right)^{*}\left(A P_{A^{k}}\right)^{*} A\right)=\mathcal{N}\left(\left(A P_{A^{k}} A^{k}\right)^{*} A\right)=\mathcal{N}\left(\left(A^{k+1}\right)^{*} A\right)
$$


where the last equality is a consequence of $P_{A^{k}}$ is an orthogonal projector onto $\mathcal{R}\left(A^{k}\right)$. Conversely, by Lemma 2.5 we have

$$
\mathcal{N}\left(\left(A^{k+1}\right)^{*} A\right) \subseteq \mathcal{N}\left(\left(\left(A^{k+1}\right)^{\dagger}\right)^{*}\left(A^{k+1}\right)^{*} A\right)=\mathcal{N}\left(\left(P_{A^{k+1}}\right)^{*} A\right)=\mathcal{N}\left(P_{A^{k}} A\right)
$$

From Theorem 2.7, $A A^{\oplus}=P_{A^{k}}$. So, from (17) it follows that

$$
\mathcal{N}\left(\left(A^{k+1}\right)^{*} A\right) \subseteq \mathcal{N}\left(A A^{\oplus} A\right) \subseteq \mathcal{N}\left(A^{\oplus} A A^{\oplus} A\right)=\mathcal{N}\left(A^{\oplus} A\right),
$$

where the last equality is due to the fact that $A^{\oplus}$ is an outer inverse.

The next result is a counterpart of [2, Theorem 3] for core EP inverses.

Theorem 3.8. Let $A \in \mathbb{C}^{n \times n}$. Then the following statements are equivalent:

(i) $A$ is $E P$;

(ii) $\left(A^{\oplus}\right)^{\oplus}=A$;

(iii) $\left(A^{\oplus}\right)^{\dagger}=A$;

(iv) $\left(A^{\dagger}\right)^{\oplus}=A$;

(v) $A P_{A}=A$.

Moreover, in this case, $A A^{\oplus}=A^{\oplus} A$ and $\left(A^{\oplus}\right)^{\dagger}=\left(A^{\dagger}\right)^{\oplus}$.

Proof. By comparing (9) and Corollary 3.6 (iii), condition (ii) is satisfied if and only if $\Sigma L=0$ and $\left((\Sigma K)^{\oplus}\right)^{\oplus}=\Sigma K$. Since $\Sigma$ is nonsingular, the former condition is equivalent to $L=0$. In consequence, from $K K^{*}+L L^{*}=I_{r}$, it then follows that $K$ is nonsingular and so $\Sigma K$ is nonsingular as well. Therefore, the condition $\left((\Sigma K)^{\oplus}\right)^{\oplus}=\Sigma K$ is always satisfied because $(\Sigma K)^{\oplus}=(\Sigma K)^{-1}$. Summarizing this reasoning, condition (ii) is equivalent to $L=0$ which holds if and only if $A$ is EP by $[2$, Lemma $1(\mathrm{v})]$.

The equivalence between (i) and (iii) follows similarly by using Corollary 3.6 (i).

On the other hand, we observe that (iv) is equivalent to $\left(\left(A^{\dagger}\right)^{\oplus}\right)^{\dagger}=A^{\dagger}$ which is equivalent to the fact that $A^{\dagger}$ is EP due to the equivalence between (i) and (iii). Since $A$ is EP if and only if $A^{\dagger}$ is EP, it follows that (iv) is equivalent to (i).

The equivalence between (i) and (v) follows from [2, Theorem 3].

Finally, since $A$ is EP then $\operatorname{Ind}(A) \leq 1$ and so $A^{\oplus}=A^{\oplus}$ by Theorem 2.8. Hence, the last assertions follow from $[2$, Theorem 3]. 
Moreover, in [2, Theorem 3] the following equivalences were proved for matrices having at most index 1:

$$
A \text { is } \mathrm{EP} \quad \Longleftrightarrow \quad A A^{\boxplus}=A^{\boxplus} A \quad \Longleftrightarrow \quad\left(A^{\boxplus}\right)^{\dagger}=\left(A^{\dagger}\right)^{\boxplus} .
$$

Nevertheless, none of these equivalences remains valid when the superscript $\bigoplus$ is replaced by $\oplus$ as we can check with the matrix

$$
A=\left[\begin{array}{ccc}
1 & -1 & 0 \\
1 & -1 & 0 \\
0 & 0 & 1
\end{array}\right]
$$

and its core EP inverse

$$
A^{\oplus}=\left[\begin{array}{lll}
0 & 0 & 0 \\
0 & 0 & 0 \\
0 & 0 & 1
\end{array}\right] \text {. }
$$

In this case, $\operatorname{Ind}(A)=2$.

\section{A weighted core EP decomposition}

In this section we give a new decomposition called weighted core EP decomposition extending the core EP decomposition from square to rectangular matrices.

Let $W \in \mathbb{C}^{n \times m}$ be a fixed nonzero matrix and $A, B \in \mathbb{C}^{m \times n}$. We define the $W$-product of $A$ and $B$ by $A \star B=A W B$, and we denote the $W$-product of $A$ with itself $\ell$ times by $A^{\star \ell}$. It is well known that if $\|A\|_{W}=\|A\|\|W\|$ then $\left(\mathbb{C}^{m \times n}, \star,\|\cdot\|_{W}\right)$ is a Banach algebra and

$$
A^{\star \ell}=(A W)^{\ell-1} A=A(W A)^{\ell-1}, \quad \ell \in \mathbb{N},
$$

where $\|\cdot\|$ denotes any (fixed but arbitrary) matrix norm on $\mathbb{C}^{m \times n}$.

Next, we establish a simultaneous unitarily upper-triangularization of a pair of rectangular matrices. We remark that this representation has no restrictions to be applied more than $W \neq 0$.

Theorem 4.1. Let $W \in \mathbb{C}^{n \times m}$ be a nonzero matrix, $A \in \mathbb{C}^{m \times n}$, and $k=\max \{\operatorname{Ind}(A W), \operatorname{Ind}(W A)\}$. Then there exist two unitary matrices $U \in \mathbb{C}^{m \times m}, V \in \mathbb{C}^{n \times n}$, two nonsingular matrices $A_{1}, W_{1} \in \mathbb{C}^{t \times t}$, and two matrices $A_{2} \in \mathbb{C}^{(m-t) \times(n-t)}$ and $W_{2} \in \mathbb{C}^{(n-t) \times(m-t)}$ such that $A_{2} W_{2}$ and $W_{2} A_{2}$ are nilpotent of indices $\operatorname{Ind}(A W)$ and $\operatorname{Ind}(W A)$, respectively, with

$$
A=U\left[\begin{array}{cc}
A_{1} & A_{12} \\
0 & A_{2}
\end{array}\right] V^{*} \text { and } W=V\left[\begin{array}{cc}
W_{1} & W_{12} \\
0 & W_{2}
\end{array}\right] U^{*}
$$


Proof. Note that $A W$ and $W A$ have the same nonzero eigenvalues (counting algebraic multiplicities). Hence, the complex Schur's decomposition ensures that the square matrices $A W$ and $W A$ can be expressed as

$$
A W=U\left[\begin{array}{cc}
C & D \\
0 & N
\end{array}\right] U^{*}, \quad W A=V\left[\begin{array}{cc}
E & F \\
0 & S
\end{array}\right] V^{*},
$$

with $C, E \in \mathbb{C}^{t \times t}$ upper-triangular nonsingular matrices, and $N \in \mathbb{C}^{(m-t) \times(m-t)}, S \in \mathbb{C}^{(n-t) \times(n-t)}$ upper-triangular with zeros on the main diagonal of both matrices. Consider the following partitions of $A$ and $W$

$$
A=U\left[\begin{array}{cc}
A_{1} & A_{12} \\
A_{21} & A_{2}
\end{array}\right] V^{*}, \quad W=V\left[\begin{array}{cc}
W_{1} & W_{12} \\
W_{21} & W_{2}
\end{array}\right] U^{*}
$$

according to the size of blocks in $A W$ and $W A$. As $N^{k}=0$, it is easy to check that

$$
\begin{aligned}
(A W)^{k} A & =U\left[\begin{array}{cc}
C^{k} & \widehat{D} \\
0 & N^{k}
\end{array}\right]\left[\begin{array}{cc}
A_{1} & A_{12} \\
A_{21} & A_{2}
\end{array}\right] V^{*}=U\left[\begin{array}{cc}
C^{k} & \widehat{D} \\
0 & 0
\end{array}\right]\left[\begin{array}{cc}
A_{1} & A_{12} \\
A_{21} & A_{2}
\end{array}\right] V^{*} \\
& =U\left[\begin{array}{cc}
C^{k} A_{1}+\widehat{D} A_{21} & C^{k} A_{12}+\widehat{D} A_{2} \\
0 & 0
\end{array}\right] V^{*}
\end{aligned}
$$

for some matrix $\widehat{D}$. Similarly, we have

$$
A(W A)^{k}=U\left[\begin{array}{ll}
A_{1} E^{k} & A_{1} \widehat{F} \\
A_{21} E^{k} & A_{21} \widehat{F}
\end{array}\right] V^{*}
$$

for some matrix $\widehat{F}$. Since $(A W)^{k} A=A(W A)^{k}$ by (18), we get $A_{21} E^{k}=0$, and so $A_{21}=0$. After a little algebra, we obtain

$$
A W=U\left[\begin{array}{cc}
A_{1} W_{1}+A_{12} W_{21} & A_{1} W_{12}+A_{12} W_{2} \\
A_{2} W_{21} & A_{2} W_{2}
\end{array}\right] U^{*}=U\left[\begin{array}{cc}
C & D \\
0 & N
\end{array}\right] U^{*}
$$

and

$$
W A=V\left[\begin{array}{cc}
W_{1} A_{1} & W_{1} A_{12}+W_{12} A_{2} \\
W_{21} A_{1} & W_{21} A_{12}+W_{2} A_{2}
\end{array}\right] V^{*}=V\left[\begin{array}{cc}
E & F \\
0 & S
\end{array}\right] V^{*} .
$$

Clearly $A_{2} W_{2}$ is nilpotent. Since $W_{1} A_{1}=E$ and $E$ is nonsingular, we have that $A_{1}$ and $W_{1}$ are both nonsingular. Furthermore, from $W_{21} A_{1}=0$ we get $W_{21}=0$. Finally, from $W_{21} A_{12}+W_{2} A_{2}=S$ we obtain that $W_{2} A_{2}$ is nilpotent.

The expressions for $A$ and $W$ found in Theorem 4.1 will be called a weighted core EP decomposition of the pair $\{A, W\}$. 
Corollary 4.2. Let $W \in \mathbb{C}^{n \times m}$ be a nonzero matrix, $A \in \mathbb{C}^{m \times n}$, and $k=\max \{\operatorname{Ind}(A W), \operatorname{Ind}(W A)\}$. We consider a weighted core EP decomposition of the pair $\{A, W\}$ as in Theorem 4.1. It then results that

(i) $(W A)^{\oplus}=(W A)_{1}^{\oplus}=V\left[\begin{array}{cc}\left(W_{1} A_{1}\right)^{-1} & 0 \\ 0 & 0\end{array}\right] V^{*}$;

(ii) $(A W)^{\oplus}=(A W)_{1}^{\oplus}=U\left[\begin{array}{cc}\left(A_{1} W_{1}\right)^{-1} & 0 \\ 0 & 0\end{array}\right] U^{*}$.

Proof. We only prove part (i) since the proof of (ii) is analogous. From Theorem 4.1 we obtain

$$
W A=V\left[\begin{array}{cc}
W_{1} A_{1} & W_{1} A_{12}+W_{12} A_{2} \\
0 & W_{2} A_{2}
\end{array}\right] V^{*} .
$$

So, a core EP decomposition of $W A$ is given by $W A=(W A)_{1}+(W A)_{2}$, where

$$
(W A)_{1}=V\left[\begin{array}{cc}
W_{1} A_{1} & W_{1} A_{12}+W_{12} A_{2} \\
0 & 0
\end{array}\right] V^{*}, \quad(W A)_{2}=V\left[\begin{array}{cc}
0 & 0 \\
0 & W_{2} A_{2}
\end{array}\right] V^{*} .
$$

Now, by applying Lemma 2.2 we get

$$
(W A)^{\oplus}=(W A)_{1}^{\oplus}=V\left[\begin{array}{cc}
\left(W_{1} A_{1}\right)^{-1} & 0 \\
0 & 0
\end{array}\right] V^{*} .
$$

\section{$5 \quad$ Weighted core EP inverses}

In this section, we introduce and study the weighted core EP inverse for rectangular matrices, extending the concept of core EP inverses.

Let $W \in \mathbb{C}^{n \times m}$ be a nonzero matrix, $A \in \mathbb{C}^{m \times n}$, and $k=\max \{\operatorname{Ind}(A W)$, $\operatorname{Ind}(W A)\}$. By using the unitary matrices $U$ and $V$ found in Theorem 4.1 corresponding to the pair $\{A, W\}$, Lemma 2.5 allows us to consider the orthogonal projectors given by

$$
P_{(A W)^{k}}=U\left[\begin{array}{cc}
I_{\operatorname{rk}\left((A W)^{k}\right)} & 0 \\
0 & 0
\end{array}\right] U^{*} \quad \text { and } \quad P_{(W A)^{k}}=V\left[\begin{array}{cc}
I_{\mathrm{rk}\left((W A)^{k}\right)} & 0 \\
0 & 0
\end{array}\right] V^{*} \text {. }
$$

Now, we consider the system given by

$$
I_{n} \star A \star X=P_{\left(I_{n} \star A^{\star k}\right)}, \quad \mathcal{R}(X) \subseteq \mathcal{R}\left(A^{\star k} \star I_{m}\right) .
$$


Theorem 5.1. If system (25) has a solution then it is unique.

Proof. Assume that $X_{1}$ and $X_{2}$ satisfy (25). As $I_{n} \star A^{\star k}=(W A)^{k}$ and $A^{\star k} \star I_{m}=(A W)^{k}$ we have

(a) $W A W X_{1}=W A W X_{2}=P_{(W A)^{k}}$,

(b) $\mathcal{R}\left(X_{1}\right) \subseteq \mathcal{R}\left((A W)^{k}\right)$ and $\mathcal{R}\left(X_{2}\right) \subseteq \mathcal{R}\left((A W)^{k}\right)$.

From (a) we get $W A W\left(X_{1}-X_{2}\right)=0$. In consequence, $\mathcal{R}\left(X_{1}-X_{2}\right) \subseteq \mathcal{N}(W A W) \subseteq \mathcal{N}(A W A W) \subseteq$ $\ldots \subseteq \mathcal{N}\left((A W)^{k}\right)$.

On the other hand, according to (b) we obtain $\mathcal{R}\left(X_{1}-X_{2}\right) \subseteq \mathcal{R}\left((A W)^{k}\right)$. So,

$$
\mathcal{R}\left(X_{1}-X_{2}\right) \subseteq \mathcal{N}\left((A W)^{k}\right) \cap \mathcal{R}\left((A W)^{k}\right)=\{0\},
$$

because $A W$ has index at most $k$. Thus, $X_{1}=X_{2}$.

When the unique matrix of Theorem 5.1 exists, it will be denoted by $A^{\oplus, W}$.

Now, we establish the existence and representation of the unique solution of the system (25) by using a weighted core EP decomposition, which has been developed for this purpose.

Theorem 5.2. The system (25) is always consistent and its unique solution is given by

$$
A^{\oplus, W}=\left(I_{n} \star A \star P_{\left(A^{\star k} \star I_{m}\right)}\right)^{\dagger}=U\left[\begin{array}{cc}
\left(W_{1} A_{1} W_{1}\right)^{-1} & 0 \\
0 & 0
\end{array}\right] V^{*} .
$$

Proof. From decomposition (19) given in Theorem 4.1 for $A$ and $W$ we have that

$$
\begin{aligned}
X & :=\left(I_{n} \star A \star P_{\left(A^{\star k} \star I_{m}\right)}\right)^{\dagger}=\left(W A W P_{(A W)^{k}}\right)^{\dagger}=\left(W A W\left[(A W)^{k}\left((A W)^{k}\right)^{\dagger}\right]\right)^{\dagger} \\
& =\left[V\left[\begin{array}{cc}
W_{1} A_{1} W_{1} & W_{1} A_{1} W_{12}+\left(W_{1} A_{12}+W_{12} A_{2}\right) W_{2} \\
0 & W_{2} A_{2} W_{2}
\end{array}\right]\left[\begin{array}{cc}
I_{\operatorname{rk}\left((A W)^{k}\right)} & 0 \\
0 & 0
\end{array}\right] U^{*}\right]^{\dagger} \\
& =\left[V\left[\begin{array}{cc}
W_{1} A_{1} W_{1} & 0 \\
0 & 0
\end{array}\right] U^{*}=U\left[\begin{array}{cc}
\left(W_{1} A_{1} W_{1}\right)^{-1} & 0 \\
0 & 0
\end{array}\right] V^{*},\right.
\end{aligned}
$$

where the projector $P_{(A W)^{k}}$ has been indicated in (24).

Now, we shall prove that the matrix $X$ satisfies the system (25). In fact, by using (24) we get

$$
\begin{aligned}
I_{n} & \star A \star X=W A W X \\
& =V\left[\begin{array}{cc}
W_{1} A_{1} W_{1} & W_{1} A_{1} W_{12}+\left(W_{1} A_{12}+W_{12} A_{2}\right) W_{2} \\
0 & W_{2} A_{2} W_{2}
\end{array}\right]\left[\begin{array}{cc}
\left(W_{1} A_{1} W_{1}\right)^{-1} & 0 \\
0 & 0
\end{array}\right] V^{*} \\
& =V\left[\begin{array}{cc}
I_{\operatorname{rk}\left((W A)^{k}\right)} & 0 \\
0 & 0
\end{array}\right] V^{*}=P_{(W A)^{k}}=P_{\left(I_{n} \star A^{\star k}\right)} .
\end{aligned}
$$


Moreover, Corollary 4.2 implies that $X=(A W)^{\oplus} A(W A)^{\oplus}$. Let $s=\operatorname{Ind}(A W)$. Since $k \geq s$, Lemma 2.3 implies that $\mathcal{R}(X) \subseteq \mathcal{R}\left((A W)^{\oplus}\right) \subseteq \mathcal{R}\left((A W)^{s}\right)=\mathcal{R}\left((A W)^{k}\right)=\mathcal{R}\left(A^{\star k} \star I_{m}\right)$. Finally, Theorem 5.1 gives the uniqueness, that is, $A^{\oplus, W}=X$.

Definition 5.3. Let $W \in \mathbb{C}^{n \times m}$ be a nonzero matrix, $A \in \mathbb{C}^{m \times n}$, and $k=\max \{\operatorname{Ind}(A W), \operatorname{Ind}(W A)\}$. The unique matrix $X \in \mathbb{C}^{m \times n}$ that satisfies system (25) is called the weighted core EP inverse of $A$.

As we have demonstrated, this matrix is $X=A^{\oplus, W}$.

Remark 5.4. When $m=n$ and $W=I_{n}$, from the representation given in Theorem 2.7 , it is easy to verify that the weighted core EP inverse and the core EP inverse are coincide.

The following result is an natural extension of Theorem 2.8 .

Corollary 5.5. Let $W \in \mathbb{C}^{n \times m}$ be a nonzero matrix, $A \in \mathbb{C}^{m \times n}$, and $k=\max \{\operatorname{Ind}(A W)$, $\operatorname{Ind}(W A)\}$ written as in (19). Then $A=A \star A^{\oplus, W} \star A$ if and only if $A_{2}=0$.

Proof. From the weighted core EP decomposition of the pair $(A, W)$ given in (19) and Theorem 5.2, we have that

$$
\begin{aligned}
A & \star A^{\oplus, W} \star A=A W A^{\oplus, W} W A \\
& =U\left[\begin{array}{cc}
A_{1} W_{1} & A_{1} W_{12}+A_{12} W_{2} \\
0 & A_{2} W_{2}
\end{array}\right]\left[\begin{array}{cc}
\left(W_{1} A_{1} W_{1}\right)^{-1} & 0 \\
0 & 0
\end{array}\right]\left[\begin{array}{cc}
W_{1} A_{1} & W_{1} A_{12}+W_{12} A_{2} \\
0 & W_{2} A_{2}
\end{array}\right] V^{*} \\
& =U\left[\begin{array}{cc}
A_{1} & A_{12}+W_{1}^{-1} W_{12} A_{2} \\
0 & 0
\end{array}\right] V^{*},
\end{aligned}
$$

whence we arrive at the conclusion that the condition $A \star A^{\oplus, W} \star A=A$ is equivalent to $A_{2}=0$.

The following result can be easily derived from Corollary 4.2 and Theorem 5.2.

Corollary 5.6. Let $W \in \mathbb{C}^{n \times m}$ be a nonzero matrix, $A \in \mathbb{C}^{m \times n}, k=\max \{\operatorname{Ind}(A W)$, $\operatorname{Ind}(W A)\}$; and consider the orthogonal projectors given in (24). Then the following assertions are true:

(i) $(A W)^{\oplus}=A^{\oplus, W} \star P_{(A W)^{k}}$;

(ii) $(W A)^{\oplus}=P_{(W A)^{k}} \star A^{\oplus, W}$;

(iii) $A^{\oplus, W}=(A W)_{1}^{\oplus} A(W A)_{1}^{\oplus}=(A W)^{\oplus} A(W A)^{\oplus}$.

The following characterization of the weighted core EP inverse is inspired in Lemma 2.3 which establishes a characterization of the core EP inverse. 
Theorem 5.7. Let $W \in \mathbb{C}^{n \times m}$ be a nonzero matrix, $A \in \mathbb{C}^{m \times n}$, and $k=\max \{\operatorname{Ind}(A W), \operatorname{Ind}(W A)\}$.

Then $X$ is the weighted core EP inverse of $A$ if and only if $X$ satisfies the conditions:

$$
X \star A^{\star(k+2)}=A^{\star(k+1)}, \quad X \star A \star X=X, \quad\left(I_{n} \star A \star X\right)^{*}=I_{n} \star A \star X, \quad \text { and } \quad \mathcal{R}(X) \subseteq \mathcal{R}\left(A^{\star k} \star I_{m}\right) .
$$

Proof. Assume that $X$ is the weighted core EP inverse of $A$, that is $X=A^{\oplus, W}$. We shall prove that the matrix $A^{\oplus, W}$ satisfies conditions (26). By definiton of the weighted core EP inverse, $\mathcal{R}(X) \subseteq$ $\mathcal{R}\left(A^{\star k} \star I_{m}\right)$ holds. According to (19) we get

$$
W A=V\left[\begin{array}{cc}
W_{1} A_{1} & W_{1} A_{12}+W_{12} A_{2} \\
0 & W_{2} A_{2}
\end{array}\right] V^{*}
$$

which implies

$$
A^{\star(k+1)}=A(W A)^{k}=U\left[\begin{array}{cc}
A_{1} & A_{12} \\
0 & A_{2}
\end{array}\right]\left[\begin{array}{cc}
\left(W_{1} A_{1}\right)^{k} & Z \\
0 & 0
\end{array}\right] V^{*}=U\left[\begin{array}{cc}
A_{1}\left(W_{1} A_{1}\right)^{k} & A_{1} Z \\
0 & 0
\end{array}\right] V^{*},
$$

where $Z=\sum_{j=0}^{k}\left(W_{1} A_{1}\right)^{j}\left(W_{1} A_{12}+W_{12} A_{2}\right)\left(W_{2} A_{2}\right)^{k-j}$. In consequence, if $G=W_{1} A_{1} W_{12}+\left(W_{1} A_{12}+\right.$ $\left.W_{12} A_{2}\right) W_{2}$, we obtain

$$
\begin{aligned}
A^{\oplus, W} & \star A^{\star(k+2)}=A^{\oplus, W} W A W\left[A(W A)^{k}\right] \\
& =U\left[\begin{array}{cc}
\left(W_{1} A_{1} W_{1}\right)^{-1} & 0 \\
0 & 0
\end{array}\right]\left[\begin{array}{cc}
W_{1} A_{1} W_{1} & G \\
0 & W_{2} A_{2} W_{2}
\end{array}\right]\left[\begin{array}{cc}
A_{1}\left(W_{1} A_{1}\right)^{k} & A_{1} Z \\
0 & 0
\end{array}\right] V^{*} \\
& =U\left[\begin{array}{cc}
I_{\mathrm{rk}\left((W A)^{k}\right)} & \left(W_{1} A_{1} W_{1}\right)^{-1} G \\
0 & 0
\end{array}\right]\left[\begin{array}{cc}
A_{1}\left(W_{1} A_{1}\right)^{k} & A_{1} Z \\
0 & 0
\end{array}\right] V^{*} \\
& =U\left[\begin{array}{cc}
A_{1}\left(W_{1} A_{1}\right)^{k} & A_{1} Z \\
0 & 0
\end{array}\right] V^{*}=A^{\star(k+1)} .
\end{aligned}
$$

Also, we have

$$
\begin{aligned}
& A^{\oplus, W} \star A \star A^{\oplus, W}=A^{\oplus, W} W A W A^{\oplus, W} \\
& =U\left[\begin{array}{cc}
\left(W_{1} A_{1} W_{1}\right)^{-1} & 0 \\
0 & 0
\end{array}\right]\left[\begin{array}{cc}
W_{1} A_{1} W_{1} & G \\
0 & W_{2} A_{2} W_{2}
\end{array}\right]\left[\begin{array}{cc}
\left(W_{1} A_{1} W_{1}\right)^{-1} & 0 \\
0 & 0
\end{array}\right] V^{*} \\
& =U\left[\begin{array}{cc}
I_{\mathrm{rk}\left((W A)^{k}\right)} & \left(W_{1} A_{1} W_{1}\right)^{-1} G \\
0 & 0
\end{array}\right]\left[\begin{array}{cc}
\left(W_{1} A_{1} W_{1}\right)^{-1} & 0 \\
0 & 0
\end{array}\right] V^{*}=A^{\oplus, W},
\end{aligned}
$$


and

$$
\begin{aligned}
\left(I_{n} \star A \star A^{\oplus, W}\right)^{*} & =\left(W A W A^{\oplus, W}\right)^{*} \\
& =\left[V\left[\begin{array}{cc}
W_{1} A_{1} W_{1} & G \\
0 & W_{2} A_{2} W_{2}
\end{array}\right]\left[\begin{array}{cc}
\left(W_{1} A_{1} W_{1}\right)^{-1} & 0 \\
0 & 0
\end{array}\right] V^{*}\right]^{*} \\
& =\left(P_{(W A)^{k}}\right)^{*}=P_{(W A)^{k}}=W A W A^{\oplus, W}=I_{n} \star A \star A^{\oplus, W} .
\end{aligned}
$$

Conversely, suppose that $X \in \mathbb{C}^{m \times n}$ satisfies (26). We assume $A$ and $W$ have the forms given in (19) and $X$ is partitioned as

$$
X=U\left[\begin{array}{cc}
X_{1} & X_{12} \\
X_{21} & X_{2}
\end{array}\right] V^{*},
$$

according to the size of blocks in $A$. Direct calculations show that the equation $X \star A^{\star(k+2)}=A^{\star(k+1)}$ is satisfied if and only if $X_{1}=\left(W_{1} A_{1} W_{1}\right)^{-1}$ and $X_{21}=0$. Thus,

$$
X=U\left[\begin{array}{cc}
\left(W_{1} A_{1} W_{1}\right)^{-1} & X_{12} \\
0 & X_{2}
\end{array}\right] V^{*} .
$$

Since $\mathcal{R}(X) \subseteq \mathcal{R}\left((A W)^{k}\right)$ can be equivalently expressed as $P_{(A W)^{k}} X=X$, it is seen that $\mathcal{R}(X) \subseteq$ $\mathcal{R}\left(A^{\star k} \star I_{m}\right)$ holds if and only if $X_{2}=0$. In consequence, by (27) we get that $\left(I_{n} \star A \star X\right)^{*}=I_{n} \star A \star X$ is equivalent to $X_{12}=0$. Hence,

$$
X=U\left[\begin{array}{cc}
\left(W_{1} A_{1} W_{1}\right)^{-1} & 0 \\
0 & 0
\end{array}\right] V^{*} .
$$

Now, Theorem 5.2 completes the proof.

\section{References}

[1] O.M. Baksalary, G. Trenkler, Revisitation of the product of two orthogonal projectors, Linear Algebra and its Applications, 430 (2009) 2813-2833.

[2] O.M. Baksalary, G. Trenkler, Core inverse of matrices, Linear and Multilinear Algebra, 58 (6) (2010) 681-697.

[3] O.M. Baksalary, G. Trenkler, On a generalized core inverse, Applied Mathematics \& Compututation, 236 (2014) 450-457.

[4] A. Ben-Israel, T.N. Greville, Generalized Inverses: Theory and Applications, Second ed., SpringerVerlag, New York, 2003. 
[5] D. Cvetković-Ilić, D. Mosić, Y. Wei. Partial orders on B(H), Linear Algebra and its Applications, 481 (2015) 115-130.

[6] M.C. Gouveia, R. Puystjens, About the group inverse and Moore-Penrose inverse of a product, Linear Algebra and its Applications, 150 (1991) 361-369.

[7] R.E. Hartwig, K. Spindelböck, Matrices for which $A^{*}$ and $A^{\dagger}$ conmmute, Linear and Multilinear Algebra, 14 (1984) 241-256.

[8] C.H. Hung, T.L. Markham, The Moore-Penrose inverse of a partitioned matrix $M=\left[\begin{array}{ll}A & B \\ C & D\end{array}\right]$, Linear Algebra and its Applications, 11 (1) (1975) 73-86.

[9] S. Malik, L. Rueda, N. Thome, Further properties on the core partial order and other matrix partial orders, Linear and Multilinear Algebra, 62, (12) (2014) 1629-1648.

[10] S.B. Malik, N. Thome, On a new generalized inverse for matrices of an arbitrary index, Applied Mathematics \& Compututation, 226 (2014) 575-580.

[11] D. Mosić, D.S. Djordjević, Weighted generalized Drazin inverse in rings, Georgian Mathematical Journal, 23 (4) (2016) 587-594.

[12] K.M. Prasad, K.S. Mohana, Core EP inverse, Linear and Multilinear Algebra, 62 (3) (2014) $792-802$.

[13] X. Wang, Core-EP decomposition and its applications, Linear Algebra and its Applications, 508 (2016) 289-300. 